\title{
Estilos parentais e o risco no uso das redes sociais em adolescentes e jovens adultos: Papel mediador da personalidade
}

\author{
Bianca Monteiro ${ }^{1}$ \& Catarina Pinheiro Mota ${ }^{1,2}$ \\ ${ }^{1}$ Universidade de Trás-os-Montes e Alto Douro \\ ${ }^{2}$ Centro de Psicologia da Universidade do Porto
}

\begin{abstract}
Resumo: De acordo com Baumrind, a relação entre a díade pais-filhos constitui-se como base da aprendizagem de competências e interações sociais. Um estilo parental baseado na assertividade e responsividade assume um papel significativo e protetor face ao risco do uso das redes sociais dos adolescentes e jovens adultos. Pretende-se analisar o efeito dos estilos parentais e da personalidade no uso das redes sociais por adolescentes e jovens adultos e testar o papel mediador da personalidade numa amostra de 936 indivíduos com idades entre os 14 e 20 anos. Os resultados apontam que o uso problemático das redes sociais é predito negativamente pelo estilo parental democrático e, positivamente pelo estilo autoritário e permissivo. Sugere-se ainda um efeito mediador negativo da personalidade emocionalmente ajustada na associação do estilo parental democrático e uso problemático das redes socias e um efeito mediador positivo entre os estilos parentais negativos e o uso problemático.
\end{abstract}

Palavras-chave: Estilos parentais; personalidade; redes sociais; adolescentes; jovens adultos.

Parental Styles and the risk on use of the social networks in adolescent and young adults: Mediational role of the personality: According to Baumrind, relationship between parents-children dyad is the base for learning skills and social interactions. A parental style based on assertiveness and responsiveness, assumes a significant and protective role regarding the dangers of social media when used by adolescents and young adults. We intend to analyze the effect of parental styles and the personality on the use of social media by adolescents and young adults, and test the mediational role of personality, in a sample of 936 individuals 14 to 20 aged. The results showed that the problematic use of social media was negatively predicted by the democratic parental style and positively predicted by the authoritarian and permissive style. Data also suggests a negative mediating effect between the emotionally adjusted personality in the democratic parental style and the problematic use of social media, and a positive mediating effect between negative parenting styles and the problematic use of social media.

Keywords: Parenting styles; personality; social networks; adolescents; young adults.

A utilização das redes sociais tem vindo a mostrar-se significativa na natureza das relações humanas e desde o seu aparecimento atraíram milhões de utilizadores que as integraram nas suas vidas diárias. Estas são um veículo atrativo de socialização que permite o estabelecimento e manutenção de relações sociais (Assunção \& Matos, 2014).

A recetividade às redes sociais é de maior relevância na adolescência, sendo este o estádio de desenvolvimento em que a preocupação com o autoconceito, com a aceitação dos pares e com a aparência física é mais significativa. Neste sentido, as redes sociais constituem-se como plataformas ideais para os jovens se conectarem aos seus pares, para facilitar a construção e expressão da identidade dentro de um contexto social, surgindo por isso a preocupação com o risco da utilização das redes sociais nesta faixa etária (Secades et al., 2014).

0 uso problemático, apesar de não ser considerado como um transtorno mental, é uma síndrome multidimensional, que se refere a comportamentos impulsivos e repetitivos que consequentemente produzem efeitos sociais, académicos ou profissionais negativos (Boubeta et al., 2015).

A teoria dos estilos parentais defendida por Baumrind $(1967,1991)$ sustenta que as famílias, como primeiro contexto de socialização, constituem-se como a base da aprendizagem de competências e interações sociais. As relações precoces têm sido identificadas como fundamentais para o desenvolvimento

${ }_{1}^{1}$ Morada para correspondência: Bianca Monteiro, Quinta dos Prados, Edifício da Escola de Ciências Humanas e Socais- Polo I, 5000-801 Vila Real, Portugal. E-mail: Bianca_monteiro95@hotmail.com.

Esta investigação é parcialmente suportada pelo Centro de Psicologia da Universidade do Porto, Fundação para a Ciência e Tecnologia (FCT UIDB/00050/2020). 
da criança, sendo a qualidade dos cuidados parentais apontada frequentemente como a variável mais importante para o desenvolvimento infantil. Neste sentido, os estilos parentais, enquanto padrão de comportamento dos pais, através de um conjunto de atitudes relacionais, comunicacionais e práticas parentais adotadas (Rinhel-Silva et al., 2012), contribuem para o desenvolvimento afetivo dos jovens e constituem-se como um fator relevante na compreensão de aspetos emocionais e comportamentais dos mesmos (Mondin, 2017).

Nesta medida, Baumrind $(1967,1991)$ propôs a classificação de três estilos parentais que se baseiam no controlo e afeto parental: o democrático, o autoritário e o permissivo. Pais que adotam o estilo democrático exercem um controlo firme, são afetuosos, calorosos e responsivos às necessidades das crianças, promovendo a autonomia e individualidade dos seus filhos. Por sua vez, pais que exercem o estilo autoritário, adotam uma posição dominante e tentam influenciar, controlar e avaliar o comportamento e atitudes dos filhos de acordo com um padrão absoluto, recorrendo de forma frequente ao uso da punição. Por fim, pais que adotam um estilo permissivo permitem que as crianças regulem o seu próprio comportamento e tomem as suas próprias decisões, impondo poucas regras de rotina, evitando tomar posições de autoridade e impor controlo ou restrições aos seus filhos.

A qualidade dos laços emocionais estabelecidos com as figuras significativas parece estar associada aos comportamentos dos adolescentes nas redes sociais, na medida em que o estilo parental democrático está associado à monitorização do comportamento dos jovens e os filhos de pais que exercem este estilo revelam menos comportamentos online de alto risco (Leung \& Lee, 2012).

Por outro lado, alguns autores (e.g., Maddahi et al., 2012) defendem que a família assume o principal suporte face ao desenvolvimento da personalidade dos jovens, considerando os estilos parentais, adotados pelos cuidadores, um dos fatores essenciais para o seu desenvolvimento e formação. Um ambiente familiar carinhoso, mais estável e com menos rivalidades tem vindo a ser relacionado positivamente a uma personalidade equilibrada nos adolescentes (Schofield et al., 2012).

A personalidade diz respeito às diferentes características e traços individuais que vão sendo desenvolvidos ao longo da vida respeitantes aos padrões de comportamento, pensamentos e sentimentos, incluindo funções próprias e que tornam o sujeito num organismo singular (Água et al., 2018).

0 modelo dos cinco fatores, também conhecido como Big Five, é considerado uma teoria explicativa e preditiva da personalidade humana e das suas relações com a conduta humana. Aglomera as tendências cognitivas, comportamentais e emocionais do indivíduo em cinco dimensões que variam ao longo de um contínuo: o neuroticismo, a extroversão, a abertura à experiência, a amabilidade e a conscienciosidade (John \& Srivastava, 1999). Deste modo, a extroversão é um traço referente a sujeitos que experimentam estados emocionais positivos, remetendo, assim, para pessoas mais sociáveis, afetuosas e otimistas. A abertura à experiência está relacionada com a flexibilidade de pensamento, fantasia, imaginação, curiosidade, criatividade, interesses culturais e abertura para novas experiências. Por sua vez, a amabilidade refere-se à capacidade do indivíduo para se relacionar com os outros. A conscienciosidade está relacionada com a capacidade de empenho, responsabilidade e honestidade. Por fim, o neuroticismo indica a tendência dos indivíduos para vivenciarem estados emocionais negativos e experimentarem uma visão negativista de si próprio e do mundo (Ross et al., 2009).

Nesta medida, os traços da personalidade parecem ser relevantes na forma como o indivíduo se reorganiza e adapta ao mundo que o rodeia, postulando-se que as características da personalidade possam também estar associadas ao comportamento dos jovens na internet, nomeadamente, à forma como os indivíduos interagem e mantêm os seus relacionamentos e como se comportam neste contexto (Pornsakulvanich, 2017).

Amichai-Hamburger e Vinitzky (2010), num estudo com 237 estudantes com uma idade média de 22 anos, concluíram que a extroversão está positivamente correlacionada com a quantidade de subscrições nas diversas redes sociais e com a interação social de um indivíduo comparativamente a indivíduos introvertidos. Por outro lado, o neuroticismo, caracterizado pela tendência a experienciar emoções negativas, encontra-se positivamente associado ao desenvolvimento de comportamentos compulsivos no uso de internet e à revelação de informação pessoal com o objetivo de autopromoção (McKinney et al., 2012). No que concerne à conscienciosidade e à amabilidade, jovens com pontuações mais elevadas nestes fatores e pontuações mais baixas no fator neuroticismo tendem a apresentar menor tendência para publicar conteúdo problemático nos seus perfis (Karl et al., 2010).

Em Portugal, há já alguns anos que têm vindo a ser desenvolvidos vários estudos no domínio do uso problemático da Internet (Assunção et al., 2017; Pontes \& Griffiths, 2015), contudo a predição dos estilos parentais e da personalidade no uso problemático das redes sociais é ainda escassa. Nesta medida, na presente investigação procurou-se compreender, para além da importância dos estilos parentais, em que medida os traços de personalidade podem assumir um papel mediador na associação entre os estilos parentais e o uso das redes sociais. 


\section{MÉTODO}

\section{Participantes}

No estudo participaram 936 adolescentes e jovens adultos portugueses de seis escolas de Ensino Secundário da Região Norte de Portugal, com idades compreendidas entre os 14 e os 20 anos ( $\mathrm{M}=16.6, D P$ = 1.190), dos quais 321 (34.3\%) são do sexo masculino e 615 (65.7\%) são do sexo feminino. A amostra da presente investigação foi do tipo probabilística, realizada de forma aleatória simples.

No que respeita à configuração familiar, 80,5\% (753) dos participantes pertence a uma família tradicional (pais, irmãos e fortuitamente avós), 15,8\% (148) apresenta configuração monoparental e apenas 3,7\% (35) reside com outros familiares (padrinhos, tios ou avós).

Relativamente ao uso das redes sociais, na presente amostra, 98,8\% (925) dos jovens são utilizadores ativos de redes sociais e $1.3 \%$ (11) não usam redes sociais. No que diz respeito ao número de contactos que os jovens têm na totalidade de contas que estão registados, $41 \%$ (384) de jovens revelam ter mais de 1000 contactos, 42,7 \% (400) afirmam ter entre 301 a 1000 contactos e apenas 16,2\% (162) participantes revelam ter 0 a 300 contactos.

\section{Instrumentos}

Foi utilizado um Questionário de Dados Sociodemográficos para recolha de dados pessoais como a idade, género, frequência de uso das redes sociais e número de contactos.

Styles \& Dimensions Questionnaire: Short Version (PSDQ; Robinson et al., 1996). Traduzido para a população portuguesa por Nunes e Mota (2013), é um questionário de autorrelato concebido para avaliar a perceção dos jovens face aos estilos parentais, separadamente. 0 questionário é constituído por 32 itens apresentados numa escala tipo Likert que varia do 1 (Nunca) ao 5 (Sempre). A organização do instrumento é elaborada em torno de três dimensões: democrático, autoritário e permissivo, estando estas dimensões divididas em diferentes subescalas: Estilo Democrático (apoio e afeto, regulação e cedência de autonomia/participação democrática), Estilo Autoritário (coerção física, hostilidade verbal e punição) e Estilo Permissivo (indulgência). Revela um Alpha de Cronbach de .88 para a figura paterna e .84 para a figura materna no que diz respeito à totalidade do instrumento. Relativamente aos valores de consistência interna para cada dimensão foram observados: Estilo Democrático (apoio e afeto .86/.82; regulação .84/.81; cedência de autonomia e participação democrática .85/.81) Estilo Autoritário (coerção física .88/.86; hostilidade verbal .59/.61; punição .73/.72) e Estilo Permissivo (Indulgência .66/.65) para o pai e para a mãe, respetivamente. No que diz respeito às análises fatoriais confirmatórias, estas apresentaram valores de ajustamento adequados tanto para o pai $\left(\chi \mathrm{i} 2(440)=200.89, p=.000 ; \chi_{\mathrm{i}}{ }^{2} / \mathrm{gl}=4.55 ; \mathrm{SRMR}=.09\right.$; $\mathrm{CFI}=.89$; RMSEA = .06) como para a mãe $\left(\chi \mathrm{i} 2(440)=1702.44 ; p=.000 ; \chi_{\mathrm{i}}{ }^{2} / \mathrm{gl}=3.87\right.$; SRMR $=.08 ; \mathrm{CFI}=.90$; RMSEA =.05).

NEO Five Factor Inventory (NEO-FFI-20; Costa \& McCrae, 1992). Traduzido e adaptado para a população portuguesa por Bertoquini e Pais-Ribeiro (2006), é uma escala de autorrelato constituída por 20 itens que avaliam os cinco fatores de personalidade: o neuroticismo ("Sinto-me, muitas vezes, desamparado(a), desejando que alguém resolva os meus problemas por mim"), a extroversão ("Muitas vezes, sinto-me a rebentar de energia"), a abertura à experiência ("Às vezes ao ler poesia e ao olhar para uma obra de arte sinto um arrepio ou uma onda de emoção"), a amabilidade ("Tendo a pensar o melhor acerca das pessoas") e a conscienciosidade ("Sou eficiente e eficaz no meu trabalho"). Os itens são respondidos numa escala tipo Likert de cinco opções de resposta que varia de "discordo fortemente (1)" a "concordo fortemente (5)". A análise da consistência interna revelou valores de alfa de Cronbach de .52 para a totalidade do instrumento. Relativamente a cada uma das dimensões, o alfa observado foi de .61 para o neuroticismo, .66 para a extroversão, .64 para a abertura a experiência, .58 para a amabilidade e .75 para a conscienciosidade. Pela análise fatorial confirmatória do instrumento verificou-se que os valores de ajustamento são adequados $\left(\chi \mathrm{i} 2(157)=546.66 ; p=.000 ; \chi_{\mathrm{i}}^{2} / \mathrm{gl}=3.48 ; \mathrm{SRMR}=.05 ; \mathrm{CFI}=.89 ; \mathrm{RMSEA}=.05\right)$.

Generalized Problematic Internet Use Scale 2 (Caplan, 2010). Adaptada para a população portuguesa por Pontes et al. (2016) é uma escala de autorrelato que avalia o uso problemático da internet com base na teoria cognitivo-comportamental de uso patológico da internet. Os utilizadores são classificados de baixo, médio e alto risco. É constituída por 15 itens em cinco subescalas: Preferência pela Interação Social Online, Regulação de Humor, Preocupação Cognitiva, Uso Compulsivo e Resultados Negativos. Os itens seguem uma escala tipo Likert-7 pontos (1= "Discordo totalmente" e 7= "Concordo Totalmente"). 0 instrumento revela um Alpha de Cronbach de .91, o que sustenta a sua utilização. No que se refere à consistência interna de cada dimensão, registaram-se os seguintes valores de alpha Cronbach: .85 para a Preferência pela 
Interação Social Online, .81 para a Regulação de Humor, .80 para a Preocupação Cognitiva, .83 para o Uso Compulsivo e .76 para os Resultados Negativos. As análises fatoriais confirmatórias indicaram os seguintes valores de ajustamento para o modelo, $\chi 2(77)=431,48 ; \chi_{\mathrm{i}}^{2} / \mathrm{gl}=5.61 ; \mathrm{p}=.001 ; \mathrm{CFI}=.95 ; \mathrm{SRMR}=.04$; RMSEA $=.07$.

\section{Procedimento}

O protocolo foi proposto e aprovado à Comissão de Ética da Universidade de Trás-os-Montes e Alto Douro e à Direção Geral dos Estabelecimentos Escolares (DGEST). Foi obtida autorização junto dos responsáveis de cada Escola e/ou Agrupamento, clarificados aspetos relativos ao objetivo e pertinência do estudo, e solicitadas autorizações para envio aos encarregados de educação. Após a aprovação, foi explicado o termo consentimento livre e esclarecido, e posteriormente procedeu-se ao preenchimento protocolar. Foi assegurada a voluntariedade da participação, bem como a garantia de confidencialidade e anonimato.

A amostra foi recolhida em seis escolas de Ensino Secundário da Região Norte de Portugal. A recolha de dados foi, assim, realizada de forma aleatória simples, tendo decorrido presencialmente em contexto de sala de aula, na presença da investigadora e dos professores responsáveis pelas diversas turmas dos vários cursos.

\section{Estratégias de análise de dados}

A análise de dados foi realizada no programa SPSS - Statistical Package for Social Sciences - na versão 23.0. Para efeitos de limpeza de dados, foram excluídos os missing values e os outliers. No que refere à estatística descritiva, foram testados os pressupostos de normalidade (medidas de assimetria e achatamento), sendo assumida a normalidade quando os valores absolutos se encontram entre -1 e 1 (Marôco, 2018). Foi analisada a informação estatística relativamente ao teste de Kolmogorov-Smirnov, os gráficos de Histogramas, Q-QPlots e Boxplot (Pallant, 2005). Procedeu-se as análises com testes paramétricos. Foram realizadas correlações, médias e desvio padrão das respetivas variáveis, análises de variância multivariada. 0 efeito moderador da regulação emocional foi testado a partir da utilização do programa AMOS (versão 24.0). Os resultados foram analisados com um valor de significância de p.<.05 (Marôco, 2014).

\section{RESULTADOS}

De acordo com o objetivo de analisar as associações entre os estilos parentais, a personalidade e o uso problemático das redes sociais em adolescentes e jovens adultos foram concretizadas análises correlacionais entre as diferentes variáveis. Os resultados das análises de correlações inter-escalas, as médias e desvios-padrão estão transcritos na Tabela 1.

Os resultados indicam a existência de correlações significativas entre as várias dimensões de estilos parentais e o uso problemático das redes sociais, nomeadamente, correlações negativas e significativas entre o apoio e afeto com as dimensões do uso problemático das redes sociais $(r=-.10$ até $r=-.16)$, regulação $(r=.09)$ e a cedência de autonomia e participação democrática $(r=-.08$ até $r=-.13)$ a ambas as figuras parentais com todas as dimensões do uso problemático das redes sociais. Por outro lado, verificase uma correlação positiva e significativa entre a coerção física $(r=.09$ até $r=.35)$, a hostilidade verbal ( $r$ $=.07$ até $r=.25)$, a punição $(r=.15$ até $r=.30)$ e a indulgência $(r=.19$ até $r=.33)$ e as dimensões do uso problemático das redes sociais.

No que diz respeito à associação entre a personalidade e o uso problemático das redes sociais, os resultados indicam existência de correlações positivas e significativas entre o neuroticismo e as dimensões do uso problemático das redes sociais $(r=.20$ até $r=.32)$. Por sua vez, foram analisadas correlações negativas e significativas entre a extroversão, abertura à experiência, amabilidade e conscienciosidade e as dimensões do uso problemático das redes sociais ( $r=-.06$ até $r=-.22$ ).

No que respeita à associação entre a personalidade e os estilos parentais, verifica-se correlação positiva entre a extroversão, abertura à experiência, amabilidade e conscienciosidade e o apoio e afeto, regulação e cedência de autonomia e participação democrática $(r=.07$ até $r=.27)$. Verifica-se, igualmente, correlação positiva entre o neuroticismo e a coerção física, hostilidade verbal, punição e indulgência a ambas figuras parentais $(r=.07$ até $r=.18)$. Observam-se também correlação negativa significativa entre o neuroticismo e o apoio e afeto, regulação e cedência de autonomia e participação democrática $(r=-.12$ ate $r=-.23$ ). Verificou-se ainda que abertura à experiência, amabilidade e conscienciosidade apresentam correlação negativa e significativa com coerção física, punição e indulgência ( $r=-.07$ até $r=-.13)$. Também a amabilidade apresenta correlação negativa e significativa com a hostilidade à mãe ( $r=-.09)$. 
Tabela 1. Correlação entre variáveis, média e desvio-padrão ( $N=936)$.

\begin{tabular}{|c|c|c|c|c|c|c|c|c|c|c|c|c|c|c|c|c|c|c|c|c|c|c|c|c|}
\hline & 1 & 2 & 3 & 4 & 5 & 6 & 7 & 8 & 9 & 10 & 11 & 12 & 13 & 14 & 15 & 16 & 17 & 18 & 19 & 20 & 21 & 22 & 23 & 24 \\
\hline \multicolumn{25}{|l|}{ Estilos Parentais- Pai } \\
\hline 1.Apoio e afeto & - & & & & & & & & & & & & & & & & & & & & & & & \\
\hline 2.Regulação & $.68^{* *}$ & & & & & & & & & & & & & & & & & & & & & & & \\
\hline $\begin{array}{l}\text { 3.Cedência de autonomia e } \\
\text { participação democrática }\end{array}$ & $.79 * *$ & $.60^{* *}$ & - & & & & & & & & & & & & & & & & & & & & & \\
\hline 4.Coerção física & $-.10^{* *}$ & $.12^{* *}$ & $-.10^{* *}$ & - & & & & & & & & & & & & & & & & & & & & \\
\hline 5.Hostilidade Verbal & $.07^{*}$ & $.44^{* *}$ & .05 & $.42^{* *}$ & - & & & & & & & & & & & & & & & & & & & \\
\hline 6.Punição & $-.11^{* *}$ & $.16^{* *}$ & $-.13^{* *}$ & $.59 * *$ & $.51^{* *}$ & - & & & & & & & & & & & & & & & & & & \\
\hline 7.Indulgência & .02 & $.16^{* *}$ & .04 & $.40^{* *}$ & $.45^{* *}$ & $.47^{* *}$ & - & & & & & & & & & & & & & & & & & \\
\hline \multicolumn{25}{|l|}{ Estilos Parentais- Mãe } \\
\hline 8.Apoio e afeto & $.66^{* *}$ & $.44^{* *}$ & $.53^{* *}$ & $-.17^{* *}$ & $-.03^{*}$ & $-.17^{* *}$ & -.03 & - & & & & & & & & & & & & & & & & \\
\hline 9.Regulação & $.51^{* *}$ & $.76^{* *}$ & $.50^{* *}$ & -.00 & $.19^{* *}$ & -.02 & .03 & $.68^{* *}$ & - & & & & & & & & & & & & & & & \\
\hline $\begin{array}{l}\text { 10.Cedência de autonomia e } \\
\text { participação democrática }\end{array}$ & $.55^{* *}$ & $.42^{* *}$ & $.78^{* *}$ & $-.14^{* *}$ & -.03 & $-.16^{* *}$ & .01 & $.72^{* *}$ & $.60^{* *}$ & - & & & & & & & & & & & & & & \\
\hline 11.Coerção física & $-.10^{* *}$ & $.08^{*}$ & $-.11^{* *}$ & $.85^{*}$ & $.36^{* *}$ & $.50^{* *}$ & $.36^{* *}$ & $-.22^{* *}$ & -.03 & $-.19^{* *}$ & - & & & & & & & & & & & & & \\
\hline 12.Hostilidade Verbal & $-.80^{*}$ & $.28^{* *}$ & $-.08^{*}$ & $.37^{* *}$ & $.78^{* *}$ & $.40^{* *}$ & $.39 * *$ & -.04 & $.20^{* *}$ & $-.07^{*}$ & $.45^{* *}$ & - & & & & & & & & & & & & \\
\hline 13.Punição & $-.20^{* *}$ & .03 & $-.18^{* *}$ & $.56^{* *}$ & $.39^{* *}$ & $.85^{* *}$ & $.41^{* *}$ & $-.22^{* *}$ & -.01 & $-.20^{* *}$ & $.60^{* *}$ & $.50^{* *}$ & - & & & & & & & & & & & \\
\hline 14.Indulgência & $-.10^{* *}$ & .05 & -.04 & $.37^{* *}$ & $.37^{* *}$ & $.41^{* *}$ & $.85^{* *}$ & -.04 & .02 & -.00 & $.38^{* *}$ & $.46^{* *}$ & $.48^{* *}$ & - & & & & & & & & & & \\
\hline \multicolumn{25}{|l|}{ Personalidade } \\
\hline 15.Neuroticismo & $-.23^{* *}$ & $-.13^{* *}$ & $-.18^{* *}$ & $.10^{* *}$ & $.07^{*}$ & $.09^{* *}$ & $.17^{* *}$ & $-.16^{* *}$ & $-.12^{* *}$ & $-.16^{* *}$ & $.11^{* *}$ & $.14^{* *}$ & $.11^{* *}$ & $.18^{*}$ & - & & & & & & & & & \\
\hline 16.Extroversão & $.19^{* *}$ & $.15^{* *}$ & $.13^{* *}$ & .04 & .06 & $.07 *$ & .03 & $.17^{* *}$ & $.17^{* *}$ & $.15^{* *}$ & .03 & .03 & .05 & .02 & $.31^{* *}$ & - & & & & & & & & \\
\hline 17.Abertura à Experiência & .04 & $.08^{*}$ & $.08^{*}$ & $-.09 * *$ & .05 & $-.10^{* *}$ & $-.07^{*}$ & $.08^{*}$ & $.07 *$ & $.11^{* *}$ & $-.10^{* *}$ & .03 & $.13^{* *}$ & $-.07 *$ & $-.09 * *$ & -.06 & - & & & & & & & \\
\hline 18.Amabilidade & $.16^{* *}$ & $.11^{* *}$ & $.14^{* *}$ & $-.07^{*}$ & -.02 & $-.09 * *$ & $-.08^{*}$ & $.13^{* *}$ & $.13^{* *}$ &, $14^{* *}$ & $-.10^{* *}$ & $-.09 * *$ & $-.13^{* *}$ & $-.12^{* *}$ & $-.20^{* *}$ & $.17^{* *}$ & .04 & - & & & & & & \\
\hline 19.Conscienciosidade & $.27^{* *}$ & $.19^{* *}$ & $.24^{* *}$ & -.05 & .04 & $-.08^{*}$ & $-.09^{* *}$ & $.23^{* *}$ & $.17^{* *}$ & $.20^{* *}$ & -.04 & .00 & -.08 & $-.11^{* *}$ & $-.18^{* *}$ & $.37^{* *}$ & $.11^{* *}$ & $.11^{* *}$ & - & & & & & \\
\hline \multicolumn{25}{|l|}{ Risco das Redes Sociais } \\
\hline $\begin{array}{l}\text { 20.Preferência pela } \\
\text { interação social }\end{array}$ & $-.11^{* *}$ & -.06 & $-.08^{*}$ & $.23^{* *}$ & $.07^{*}$ & $.22^{* *}$ & $.25^{* *}$ & $-.13^{* *}$ & $-.09^{* *}$ & $-.09^{* *}$ & $.18^{* *}$ & $.07^{*}$ & $.22^{* *}$ & $.26^{* *}$ & $.19^{* *}$ & $-.15^{* *}$ & $-.12^{* *}$ & -.05 & $-.13^{* *}$ & - & & & & \\
\hline 21.Regulação de Humor & $-.13^{* *}$ & -.02 & -.05 & $.11^{* *}$ & $.11^{* *}$ & $.15^{* *}$ & $.19^{* *}$ & $-.08^{*}$ & -.01 & -.04 & $.09^{* *}$ & $.16^{* *}$ & $.16^{* *}$ & $.20^{* *}$ & $.26^{* *}$ & $-.10^{* *}$ & -.02 & $-.06^{*}$ & $-.08^{*}$ & $.47^{*}$ & - & & & \\
\hline 22.Preocupação Cognitiva & $-.11^{* *}$ & .02 & $-.08^{*}$ & $.26^{* *}$ & $.20^{* *}$ & $.29^{* *}$ & $.33^{* *}$ & $-.13^{* *}$ & -.05 & $-.11^{* *}$ & $.23^{* *}$ & $.23^{* *}$ & $.29^{* *}$ & $.31^{* *}$ & $.29^{* *}$ & -.05 & $-.07^{*}$ & $-.08^{*}$ & $-.11^{* *}$ & $.50^{* *}$ & $.50^{* *}$ & - & & \\
\hline 23.Uso compulsivo & $-10^{* *}$ & .01 & $-.09^{* *}$ & $.22^{* *}$ & $.21^{* *}$ & $.23^{* *}$ & $.32^{* *}$ & $-.10^{* *}$ & -.02 & $-.11^{* *}$ & $.21^{* *}$ & $.25^{* *}$ & $.26^{* *}$ & $.31^{* *}$ & $.25^{* *}$ & -.03 & $-.07^{*}$ & $-.07^{*}$ & $-.17^{* *}$ & $.40^{* *}$ & $.42^{* *}$ & $.76^{* *}$ & - & \\
\hline 24.Resultados negativos & $-.16^{* *}$ & .02 & $-.12^{* *}$ & $.35^{* *}$ & $.20^{* *}$ & $.30^{* *}$ & $.33^{* *}$ & $-.17^{* *}$ & -.02 & $-.13^{* *}$ & $.31^{* *}$ & $.23^{* *}$ & $.30^{* *}$ & $.32^{* *}$ & $.29^{* *}$ & $-.10^{* *}$ & -.04 & $-.07^{*}$ & $-.22^{* *}$ & $.46^{* *}$ & $.40^{* *}$ & $.62^{* *}$ & $.65^{* *}$ & - \\
\hline M & 2.52 & 3.19 & 3.38 & 1.39 & 2.67 & 1.96 & 2.05 & 3.89 & 3.53 & 3.58 & 1.45 & 2.85 & 2.05 & 2.17 & 2.74 & 3.74 & 2.98 & 3.31 & 3.88 & 2.22 & 3.46 & 2.42 & 2.86 & 1.99 \\
\hline DP & .74 & .93 & .98 & .74 & .84 & .84 & .78 & .89 & .93 & .87 & .76 & .85 & .86 & .80 & .77 & .70 & .85 & .77 & .60 & 1.38 & 1.70 & 1.39 & 1.60 & 1.24 \\
\hline
\end{tabular}


Variância dos estilos parentais, personalidade e risco do uso das redes sociais

Para a análise das diferenças dos estilos parentais, personalidade e risco do uso das redes sociais em função das dimensões sociodemográficas, foram realizadas várias análises de comparação de médias (teste-t para amostras independentes) e análise de variância multivariada (MANOVA).

Para testar as diferenças entre os estilos parentais, personalidade e uso problemático das redes sociais em função da variável número de contactos/seguidores nas redes sociais, procedeu-se à sua categorização em três níveis ( 0 a 300 pessoas, 301 a 1000 pessoas, mais de 1000 pessoas).

No que respeita aos estilos parentais do pai, as análises diferenciais apontam para diferenças significativas em função do número de contactos/seguidores, $F(14,186)=1.84, p=.028, \eta^{2}=.93$, nomeadamente, na regulação, $F(2,933)=3.47 p=.031, \eta^{2}=.65$, cedência de autonomia e participação democrática, $F(2,933)=4.57, p=.011, \eta^{2}=.78$, e indulgência, $F(2,933)=3.57, p=.028, \eta^{2}=.66$. Tendo em conta comparações post-hoc, de acordo com o teste de Scheffe, os indivíduos que têm 301 a 1000 contactos tendem a revelar maior perceção de regulação e cedência de autonomia e participação democrática face ao pai em comparação com o grupo que apresenta mais de 1000 contactos. Por seu turno, o grupo de jovens que aponta ter mais de 1000 contactos manifesta maior perceção de indulgência do pai em comparação com o grupo que indica ter 0 a 300 contactos e, ainda, com o grupo que aponta possuir 301 a 1000 contactos. Relativamente aos estilos parentais da mãe, não foram observadas diferenças significativas em função do número de contactos/seguidores, $F(14,186)=1.63, p=.065, \eta^{2}=.85$ (Tabela 2).

As análises multivariadas apontam, também, para a presença de diferenças estatisticamente significativas da personalidade em função do número de contactos/seguidores, $F(10,1860)=4.59, p=$ $<.001, \eta^{2}=1.0$, nomeadamente nas dimensões neuroticismo, $F(2,933)=4.03, p=.018, \eta^{2}=.72$, extroversão, $F(2,933)=6.90, p=.001, \eta^{2}=.92$, e amabilidade, $F(2,933)=3.71, p=.025, \eta^{2}=.68$. Assim, observou-se que os indivíduos com mais de 1000 contactos tendem a revelar maiores níveis de neuroticismo em comparação com o grupo de 301 a 1000 contactos. Da mesma forma, o grupo de jovens com mais de 1000 contactos indicou maiores níveis de extroversão quando comparado com o grupo de 0 a 300 contactos e ainda com o grupo de 301 a 1000 contactos. Por seu turno, os jovens que têm 301 a 1000 contactos apresentam maiores pontuações de amabilidade quando comparados com jovens que têm mais de 1000 contactos.

No que toca ao uso problemático das redes sociais em função do número de contactos/ seguidores, as análises multivariadas apontam, igualmente, para a presença de diferenças estatisticamente significativas, $F(10,18)=2,94, p=.001, \eta^{2}=.98$, nomeadamente, nas variáveis preferência pela interação social online, $F(2,933)=3.99, p=.019, \eta 2=.72$, preocupação cognitiva, $F(2,933)=8.84, p=<.001, \eta 2=.97$, uso compulsivo $F(2,933)=10.09, p=<.001, \eta 2=.99$, e resultados negativos, $F(2,933)=3.60, p=.028, \eta 2=$ .67. Os resultados observados permitem constatar que os jovens que referem ter mais de 1000 contactos indicam maiores pontuações de preferência pela interação social online e resultados negativos em comparação com os jovens que têm 301 a 1000 contactos. Do mesmo modo, os jovens que indicam ter mais de 1000 contactos apresentam maiores níveis de preocupação cognitiva e uso compulsivo quando comparados com o grupo que tem 0 a 300 contactos e ainda com o grupo de 301 a 1000 contactos e uso compulsivo (Tabela 2).

\section{Efeito preditivo dos estilos parentais no risco do uso das redes sociais: papel mediador da personalidade}

Com o objetivo de perceber o efeito preditivo dos estilos parentais positivos (estilo democrático) e estilos parentais negativos (estilo autoritário e estilo permissivo) no uso problemático das redes sociais, bem como o efeito mediador dos traços de personalidade emocionalmente ajustados (extroversão, abertura à experiencia, amabilidade e conscienciosidade) e da personalidade negativa (neuroticismo) desempenha nesta associação, realizou-se um modelo de equações estruturais, através do programa AMOS.

Neste sentido, inicialmente, constatam-se vários efeitos de predição, nomeadamente, o estilo parental positivo (estilo democrático) da figura paterna prediz negativamente o uso problemático das redes sociais $(\beta=-.13)$ e, por seu turno, os estilos parentais negativos (estilo autoritário e permissivo) ( $\beta=$ .44) predizem positivamente o uso problemático das redes sociais. Da mesma forma, observa-se que o estilo parental positivo (estilo democrático) prediz positivamente a traços da personalidade emocionalmente ajustados $(\beta=.41)$ e negativamente o neuroticismo $(\beta=-.23)$ e, ainda que os estilos parentais negativos (estilo autoritário e permissivo) predizem positivamente o neuroticismo $(\beta=.14)$. Por sua vez, foi possível observar que traços da personalidade emocionalmente ajustados predizem negativamente o uso problemático das redes sociais $(\beta=-.12)$ e o neuroticismo prediz positivamente o uso problemático das redes sociais $(\beta=.25)$. 
Tabela 2. Análise diferencial dos estilos parentais, personalidade e risco do uso das redes sociais em função do número contactos/seguidores nas redes sociais.

\begin{tabular}{|c|c|c|c|c|}
\hline & $\begin{array}{l}\text { Número de } \\
\text { contactos/seguidores }\end{array}$ & $M \pm D P$ & IC95\% & $\begin{array}{l}\text { Direção das } \\
\text { diferenças }\end{array}$ \\
\hline \multicolumn{5}{|l|}{$\begin{array}{c}\text { Estilos parentais } \\
\text { Pai }\end{array}$} \\
\hline \multirow{3}{*}{$\begin{array}{c}\text { Regulação } \\
\text { (E. democrático) }\end{array}$} & $1-0$ a 300 & $3.21 \pm .89$ & {$[3.06,3.35]$} & \multirow{3}{*}{$2>3$} \\
\hline & $2-301$ a 1000 & $3.27 \pm .87$ & {$[3.18,3.36]$} & \\
\hline & 3- mais de 1000 & $3.10 \pm .99$ & {$[3.00,3.19]$} & \\
\hline \multirow{3}{*}{$\begin{array}{l}\text { Cedência de } \\
\text { autonomia e } \\
\text { participação } \\
\text { democrática } \\
\text { (E. democrático) }\end{array}$} & $1-0$ a 300 & $3.43 \pm .94$ & {$[3.27,3.58]$} & \multirow{3}{*}{$2>3$} \\
\hline & 2- 301 a 1000 & $3.47 \pm .90$ & {$[3.38,3.57]$} & \\
\hline & 3- mais de 1000 & $3.27 \pm 1.05$ & {$[3.17,3.37]$} & \\
\hline \multirow{3}{*}{$\begin{array}{l}\text { Indulgência } \\
\text { (E. permissivo) }\end{array}$} & $1-0$ a 300 & $1.89 \pm .67$ & {$[1.77,2.02]$} & \multirow{3}{*}{$3>1 ; 2>1$} \\
\hline & $2-301$ a 1000 & $2.08 \pm .79$ & {$[2.00,2.16]$} & \\
\hline & 3- mais de 1000 & $2.08 \pm .82$ & {$[2.00,2.16]$} & \\
\hline \multicolumn{5}{|l|}{ Personalidade } \\
\hline \multirow{3}{*}{ Neuroticismo } & $1-0$ a 300 & $2.67 \pm .84$ & {$[2.55,2.80]$} & \multirow{3}{*}{$3>2$} \\
\hline & 2- 301 a 1000 & $2.68 \pm .73$ & {$[2.61,2.76]$} & \\
\hline & 3- mais de 1000 & $2.83 \pm .79$ & {$[2.75,2.90]$} & \\
\hline \multirow{3}{*}{ Extroversão } & $1-0$ a 300 & $3.60 \pm .73$ & {$[3.49,3.71]$} & \multirow{3}{*}{$3>1 ; 3>2$} \\
\hline & 2- 301 a 1000 & $3.71 \pm .68$ & {$[3.64,3.76]$} & \\
\hline & 3- mais de 1000 & $3.83 \pm .70$ & {$[3.76,3.90]$} & \\
\hline \multirow{3}{*}{ Amabilidade } & $1-0$ a 300 & $3.26 \pm .85$ & {$[3.14,3.38]$} & \multirow{3}{*}{$2>3$} \\
\hline & 2- 301 a 1000 & $3.39 \pm .72$ & {$[3.31,3.46]$} & \\
\hline & 3- mais de 1000 & $3.25 \pm .78$ & {$[3.17,3.33]$} & \\
\hline \multicolumn{5}{|l|}{$\begin{array}{l}\text { Uso problemático } \\
\text { das Redes Sociais }\end{array}$} \\
\hline \multirow{3}{*}{$\begin{array}{l}\text { Preferência pela } \\
\text { Interação social } \\
\text { online }\end{array}$} & 1- 0 a 300 & $2.07 \pm .1 .29$ & {$[1.85,2.29]$} & \multirow{3}{*}{$3>2$} \\
\hline & 2- 301 a 1000 & $2.13 \pm 1.29$ & {$[1.99,2.26]$} & \\
\hline & 3- mais de 1000 & $2.37 \pm 1.49$ & {$[2.23,2.50]$} & \\
\hline \multirow{3}{*}{$\begin{array}{l}\text { Preocupação } \\
\text { Cognitiva }\end{array}$} & $1-0$ a 300 & $2.13 \pm 1.20$ & {$[1.91,2.34]$} & \multirow{3}{*}{$3>1 ; 3>2$} \\
\hline & 2- 301 a 1000 & $2.33 \pm 1.32$ & {$[2.19,2.46]$} & \\
\hline & 3- mais de 1000 & $2.63 \pm 1.49$ & {$[2.49,2.77]$} & \\
\hline \multirow{3}{*}{ Uso compulsivo } & 1- 0 a 300 & $2.48 \pm 1.48$ & {$[2.23,2.73]$} & \multirow{3}{*}{$3>1 ; 3>2$} \\
\hline & 2- 301 a 1000 & $2.76 \pm 1.53$ & {$[2.60,2.92]$} & \\
\hline & 3- mais de 1000 & $3.11 \pm 1.68$ & {$[2.95,3.27]$} & \\
\hline \multirow{3}{*}{$\begin{array}{l}\text { Resultados } \\
\text { Negativos }\end{array}$} & $1-0$ a 300 & $1.93 \pm 1.19$ & {$[1.74,2.13]$} & \multirow{3}{*}{$3>2$} \\
\hline & 2- 301 a 1000 & $1.88 \pm 1.16$ & {$[1.76,2.00]$} & \\
\hline & 3- mais de 1000 & $2.11 \pm 1.32$ & {$[1.99,2.24]$} & \\
\hline
\end{tabular}

Após introdução da variável mediadora e através do método Bootstrap, observou-se que o efeito direto inicial do estilo parental positivo (estilo democrático) sobre o uso problemático das redes sociais perdeu magnitude $\left(\beta_{\text {inicial-EstilosPositivos }}=-.13 ; \beta_{\text {final-EstilosPositivos }}=-.02\right)$ e significância $\left(p_{\text {inicial }}=<.001 ; p_{\text {final }}=.54\right)$, observando-se uma mediação total, no qual os traços da personalidade emocionalmente ajustados assumem uma mediação total negativa, $\beta=-.05$; IC 90\% [-.14, -.06].

Verifica-se ainda que o efeito dos estilos parentais negativos (estilo autoritário e permissivo) sobre o uso problemático das redes sociais perdeu magnitude $\left(\beta_{\text {inicial-EstilosNegatvios }}=.44 ; \beta_{\text {final-EstilosNegatvios }}=.40\right)$, no qual a personalidade negativa assume uma mediação parcial positiva, $\beta=.03$; IC 90\% $[.02$, .06] na associação entre os estilos parentais negativos (estilo autoritário e permissivo) e o uso problemático das redes sociais (figura 1 ).

0 modelo apresenta os índices de ajustamento adequados, $\chi_{i}^{2}(111)=949.92, p=.000, \chi_{i}^{2} / g \mathrm{l}=8.56$; $\mathrm{CFI}=.86, \mathrm{GFI}=.90, \mathrm{RMR}=.07, \mathrm{AGFI}=.86, \mathrm{NFI}=.84, \mathrm{IFI}=.86, \mathrm{RMSEA}=.09$. 


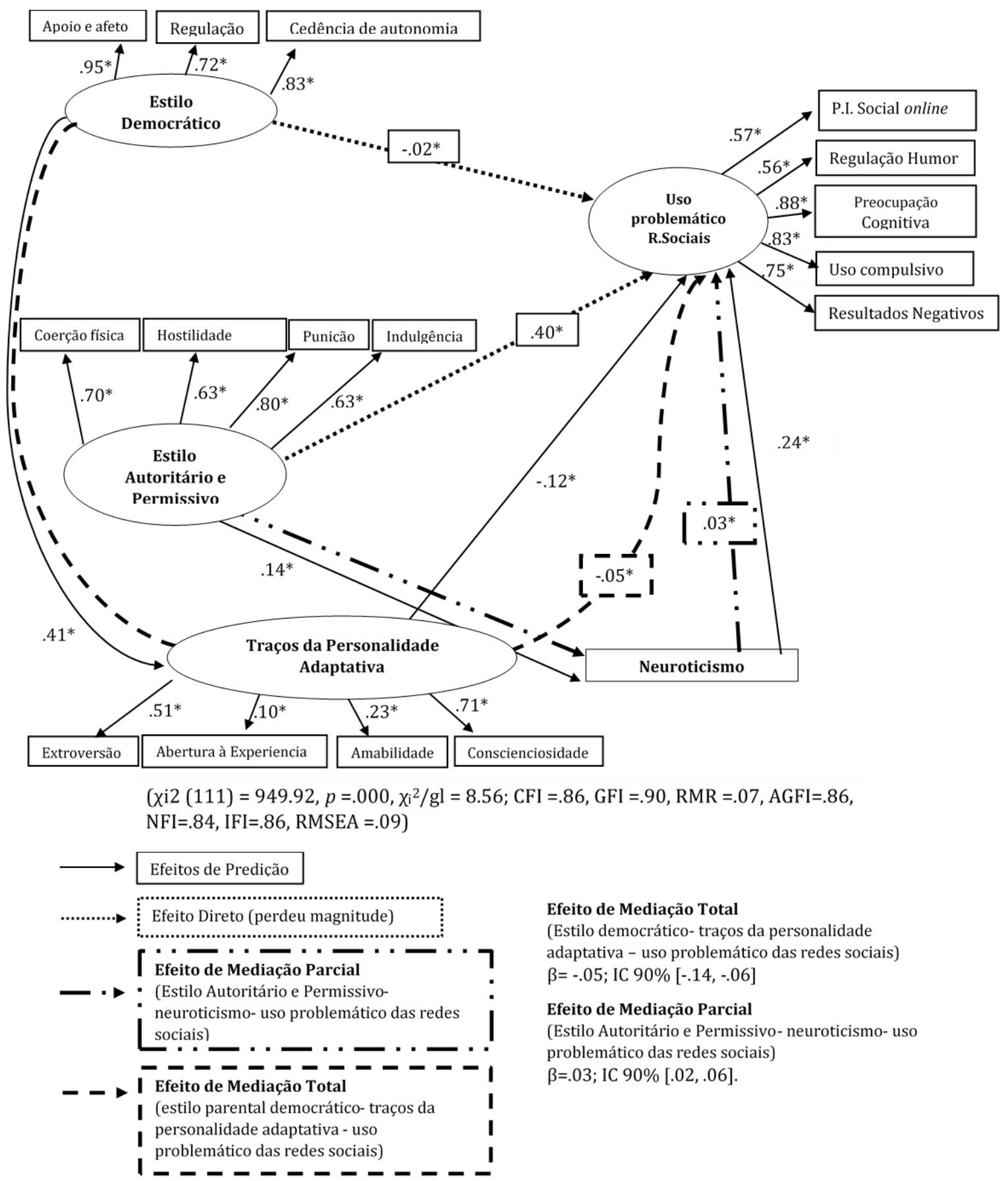

Figura 1. Modelo representativo do efeito mediador da personalidade na associação entre os estilos parentais da figura paterna e o risco do uso das redes sociais.

No que diz respeito à figura materna (Figura 2.), inicialmente, verificou-se uma predição inicial positiva dos estilos parentais negativos (estilo autoritário e permissivo) no desenvolvimento do uso problemático das redes sociais $(\beta=.42)$. Observa-se, também, que o estilo parental positivo (estilo democrático) prediz positivamente os traços da personalidade emocionalmente ajustados $(\beta=.38) \mathrm{e}$ negativamente o neuroticismo $(\beta=-.16)$. Por sua vez, verificou-se que os estilos parentais negativos (estilo autoritário e permissivo) predizem positivamente o neuroticismo $(\beta=.15)$. Verificou-se, ainda, que os traços da personalidade emocionalmente ajustados predizem negativamente $(\beta=-12)$ o uso problemático das redes sociais e o neuroticismo $(\beta=.24)$ prediz positivamente o uso problemático das redes sociais.

Após introdução da variável mediadora e através do método Bootstrap observou-se que o efeito direto inicial dos estilos parentais negativos (estilo autoritário e permissivo) sobre o uso problemático das redes sociais perdeu magnitude $\left(\beta_{\text {inicial-EstilosNegativos }}=.43 ; \beta_{\text {final-EstilosNegativos }}=.39\right)$, observando-se uma mediação parcial positiva, $\beta=.03$; IC $90 \%[.03, .10]$, no qual o neuroticismo assume uma mediação parcial 
positiva na associação entre os estilos parentais negativos (estilo autoritário e permissivo) e o uso problemático das redes sociais.

Verificam-se, ainda, efeitos de mediação total, sendo que tanto os traços da personalidade emocionalmente ajustados, $\beta=-.04$; IC 90\% [-.14, -.04], como o neuroticismo, $\beta=-.04$; IC 90\% [-.14, -.04], assumem uma mediação total negativa na associação entre o estilo parental positivo (estilo democrático) e o uso problemático das redes sociais.

0 modelo apresenta os índices de ajustamento adequados, $\chi_{\mathrm{i}}^{2}(111)=772.19, p=.000, \chi^{2} / g l=6.96$; $\mathrm{CFI}=.88, \mathrm{GFI}=.91, \mathrm{RMR}=.06, \mathrm{AGFI}=.88, \mathrm{NFI}=.86, \mathrm{IFI}=.88, \mathrm{RMSEA}=.08$.

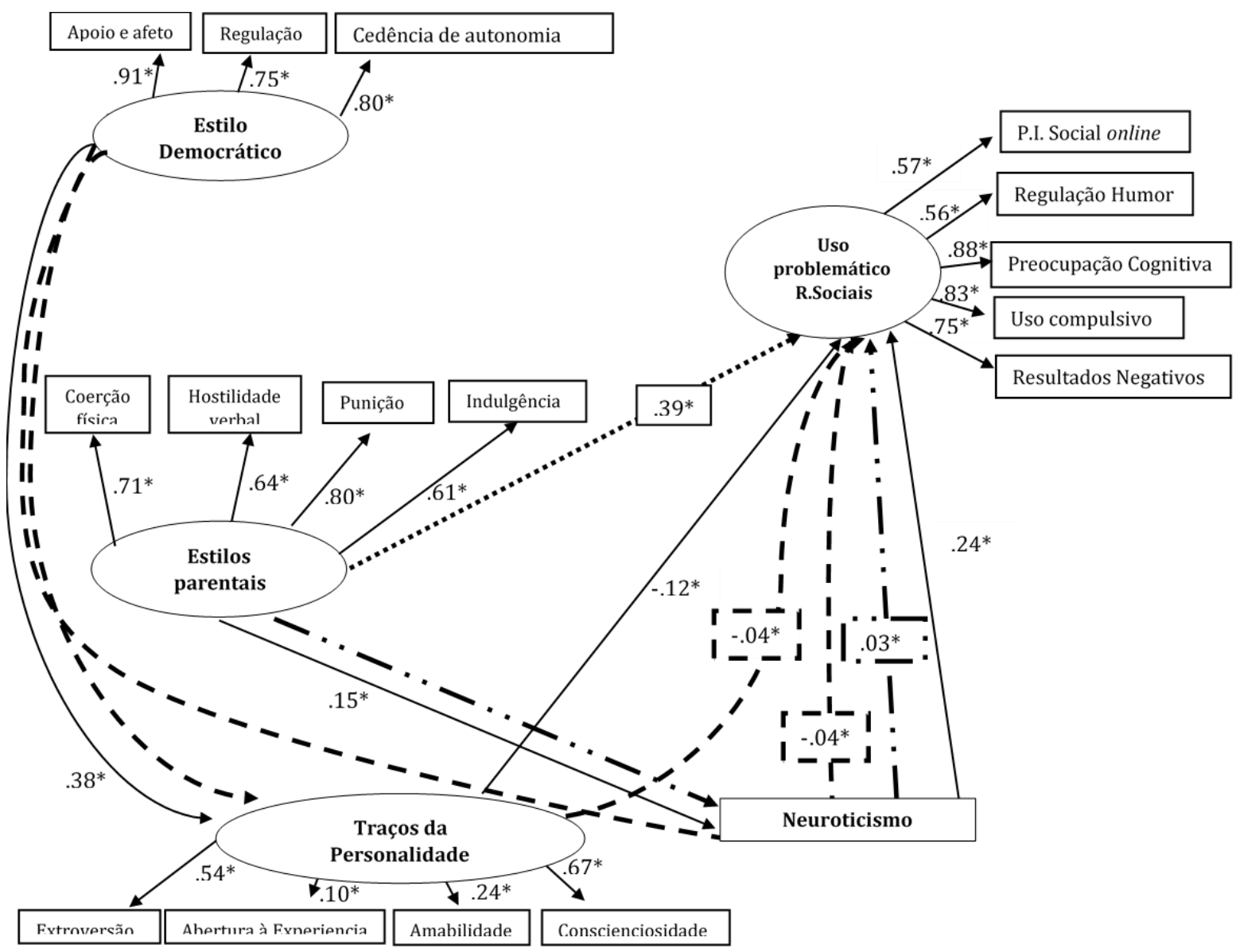

$\left(\chi \mathrm{i} 2(111)=772.19, p=.000, \chi_{\mathrm{i}}^{2} / \mathrm{gl}=6.96 ; \mathrm{CFI}=.88, \mathrm{GFI}=.91, \mathrm{RMR}=.06\right.$, $A C F I=88 \mathrm{NFI}=86 \mathrm{IFI}=88$ RMSFA $=08 \mathrm{I}$

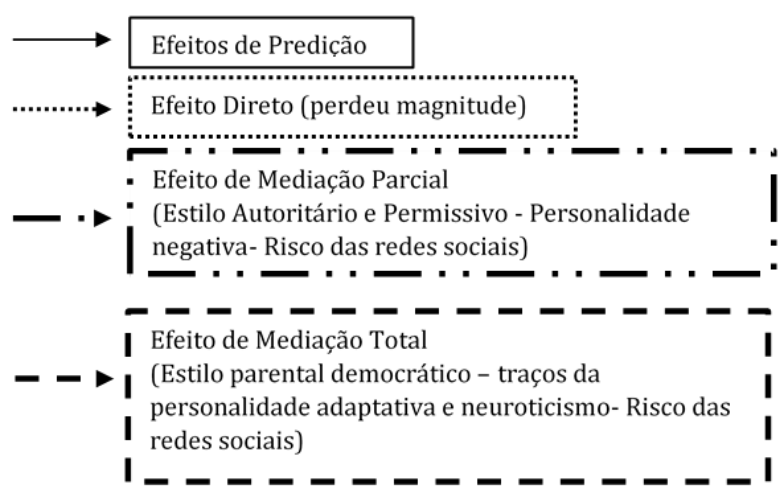

\section{Efeito de Mediação Total}

(Estilo parental democrático- traços da personalidade adaptativa - uso problemático das redes sociais) $\beta=-.04 ;$ IC $90 \%[-.14,-.04]$

Efeito de Mediação Total

(Estilo parental democrático - neuroticismo- uso problemático das redes sociais)

$\beta=-.04 ;$ IC $90 \%[-.14,-.04]$

\section{Efeito de Mediação Parcial}

(Estilo Autoritário e Permissivo- neuroticismo - uso problemático das redes sociais)

$\beta=.03$; IC $90 \%[.03, .10]$

Figura 2. Modelo representativo do efeito mediador da personalidade na associação entre os estilos parentais da figura materna e o risco do uso das redes sociais. 
Estilos parentais, personalidade e o risco no uso das redes sociais

\section{DISCUSSÃO}

0 presente estudo teve como principal objetivo analisar o papel dos estilos parentais e da personalidade no desenvolvimento de comportamentos de risco no uso das redes sociais em adolescentes e jovens adultos.

Os resultados obtidos indicam que o estilo democrático está correlacionado negativamente com o risco do uso das redes sociais e que, por sua vez, os estilos autoritário e permissivo se associam positivamente com o risco do uso das redes sociais. Contrariamente, quer uma posição autoritária, quer indulgente parece assumir-se como um fator de risco para o uso problemático das redes sociais e equitativamente a uma maior exposição aos riscos advindos do seu uso. Os resultados obtidos vão ao encontro de estudos anteriores (Chou \& Lee, 2017; Dogan et al., 2015) que referem que os estilos parentais são um preditor significativo do uso problemático da internet e que o estilo autoritário e indulgente dos pais está associado ao uso problemático da internet. Corrobora que os adolescentes que percecionam as figuras parentais como mais calorosas, amorosas, com menor rejeição e punição evidenciam menor probabilidade de desenvolverem comportamentos psicossociais negativos ou problemáticos, ou mesmo dependência da internet.

A partir da análise dos dados foi possível, ainda, verificar que o neuroticismo se associa positivamente com o uso problemático das redes sociais e, que, por sua vez, a extroversão, abertura à experiência, amabilidade e conscienciosidade se associa negativamente com o uso problemático das redes sociais. Os dados obtidos vão ao encontro do que é expectável, uma vez que o uso problemático das redes sociais está correlacionado positivamente com características da personalidade que refletem um ajustamento emocional desajustado (Ross et al., 2009). Este estudo vai ao encontro dos resultados dos estudos anteriores (e.g., Giota \& Kleftaras, 2013; Lo, 2019; Song et al., 2014) que salientam que o neuroticismo surge positivamente associado a um maior uso de redes sociais e de mensagens instantâneas para combaterem a solidão.

Relativamente à associação entre os estilos parentais e a personalidade, os resultados indicam que o estilo democrático se associa positivamente com a extroversão, abertura à experiência, amabilidade e conscienciosidade e negativamente com o neuroticismo. Por sua vez, os estilos autoritário e permissivo encontram-se associados positivamente ao neuroticismo e negativamente com a abertura à experiência, amabilidade e conscienciosidade. Também, a hostilidade à mãe se associa negativamente com a amabilidade. Assim, os resultados parecem indicar que os jovens que vivenciam um ambiente familiar responsivo tendem a desenvolver modelos internos dinâmicos positivos (Bowlby, 1969) que promovem um funcionamento saudável e competente em vários domínios da vida do adolescente (Schofield et al., 2012). Contrariamente, atitudes parentais hostis ou coercitivas propiciam a inibição do desenvolvimento pró-social dos jovens e tornam-se fatores de risco para o desenvolvimento de problemas comportamentais relacionando-se com comportamentos opositores e agressivos e com défices na autorregulação (Anaya \& Pérez-Edgar, 2018). Estes resultados são consistentes com a literatura (Tur-Porcar, 2017; Yazdani \& Daryei, 2016; Zhou et al., 2017) que destaca a existência de relação entre o estilo democrático e o ajustamento psicossocial do adolescente.

No que respeita ao número de contactos/seguidores, foi passível de se verificar que os jovens que têm 301 a 1000 contactos tendem a revelar perceção de maior regulação e cedência de autonomia e participação democrática, e que o grupo de jovens que aponta ter mais de 1000 contactos manifesta perceção de maior indulgência do pai. Novamente se verifica que uma estrutura familiar calorosa e responsiva permite aos adolescentes um maior controlo na sua utilização e na seleção dos "contactos ou seguidores" e que, por seu turno, um estilo parental permissivo, que está relacionado com a autorregulação comportamental do adolescente, com a ausência de regras, controlo ou restrições (Baumrind 1967, 1991). Adolescentes que revelam dificuldades de regulação emocional tendem a utilizar a comunicação online para a criação de relacionamentos com pessoas desconhecidas (Dogan et al., 2015).

Foram ainda encontradas diferenças do número de contactos/seguidores face à personalidade, verificando-se que os indivíduos com mais de 1000 contactos tendem a revelar maiores níveis de neuroticismo e extroversão e que os jovens que têm 301 a 1000 contactos apresentam maiores pontuações de amabilidade. Os resultados vão ao encontro de alguns estudos (e.g., Ross et al., 2009; Wilson et al., 2009) que referem que em consistência com o contacto real, jovens extrovertidos procuram contacto social virtual e estão mais envolvidos durante a experiência social online em comparação com jovens introvertidos. Isto poderá ser justificado pelo facto de jovens mais extrovertidos serem impulsionados pelo desejo de interação, enquanto o neuroticismo tende a ser o traço que identifica aqueles que agem frequentemente com o objetivo de se auto engrandecerem (Gosling et al., 2011). No que respeita ao traço amabilidade, os autores Vlachopoulou e Boutsouki (2014) mostraram que jovens com maiores pontuações 
neste traço tendem a usar as redes sociais como uma ferramenta de socialização online e para troca de informações com outros utilizadores.

Ainda no que se refere ao número de contactos/seguidores, os resultados apontam para diferenças significativas no uso problemático, sendo que os indivíduos que referem ter mais de 1000 contactos indicam maiores pontuações de preferência pela interação social online e resultados negativos, preocupação cognitiva e uso compulsivo. Tal facto poderá ser explicado porque quanto maior for a rede de contactos online, menor é o conhecimento real de cada indivíduo adicionado, o que pode aumentar a probabilidade de exposição aos riscos, como, por exemplo o assédio e roubo da identidade. E, por outro lado, maior é a motivação para a socialização online, o que pode levar ao uso compulsivo e, consequentemente, à adição.

Por fim, cabe ressaltar que, tal como delineado nas hipóteses, o estilo parental autoritário e permissivo de ambas as figuras parentais predizem positivamente o uso problemático das redes socias nos adolescentes e jovens adultos. Este resultado vai ao encontro do expectável, dado que os jovens que vivenciam um estilo parental autoritário vivenciam ambientes com baixo calor emocional, reduzida troca de afeto, distância das figuras parentais e os que vivenciam um ambiente permissivo têm liberdade na tomada das suas próprias decisões e têm por hábito não serem contrariados.

Por sua vez, foi ainda possível verificar que o estilo parental democrático prediz de forma significativa e negativa o uso problemático das redes socias nos adolescentes e jovens adultos. 0 resultado obtido era, de certa forma, esperado, sendo que um ambiente responsivo e caloroso influencia positivamente a forma como eles aprendem e se relacionam com os outros, assim como na regulação dos comportamentos. Outros estudos realizados anteriormente (Chou \& Lee, 2017; Dogan et al., 2015) vão ao encontro destes resultados salientando que o estilo parental é um preditor significativo no comportamento dos jovens na exploração do mundo que os rodeia, nomeadamente, no uso das redes sociais.

Os resultados de predição indicam, ainda, que a prática de um estilo parental democrático de ambas as figuras parentais predizem positivamente traços positivos da personalidade que espelham uma emocionalidade ajustada e, por seu turno, predizem negativamente o traço de neuroticismo. Por sua vez, verificou-se que os estilos autoritário e permissivo predizem positivamente a presença de neuroticismo. Tais resultados vão ao encontro do supracitado na revisão de literatura que subentende que um estilo parental democrático propicia o desenvolvimento de uma estrutura de personalidade emocionalmente mais ajustada (Schofield et al., 2012). Contrariamente, o estilo parental autoritário ou permissivo pode recriar ambientes parentais negativos ao não atender às necessidades primordiais dos filhos, podendo propiciar o desenvolvimento desajustado dos jovens. Yazdani e Daryei (2016), num estudo com 233 participantes, sublinharam a associação positiva entre um ambiente parental desajustado e o desenvolvimento desequilibrado dos jovens.

Através da análise do papel mediador da personalidade foi possível concluir que os traços da personalidade emocionalmente ajustados assumem uma mediação total negativa na associação entre o estilo parental democrático e o uso problemático das redes sociais. Neste sentido, observou-se que o estilo parental democrático prediz positivamente as características de personalidade emocionalmente ajustadas e que estas predizem negativamente o uso problemático das redes sociais. Portanto, podemos concluir que, na presente amostra as características da personalidade emocionalmente ajustadas exercem um fator protetor no uso problemático das redes sociais em adolescentes e jovens adultos. Desta feita, o presente resultado parece enfatizar a predição negativa dos traços da personalidade emocionalmente ajustados no desenvolvimento de comportamentos de uso problemático das redes sociais, assumindo os traços da personalidade emocionalmente ajustados um efeito condutor negativo na associação entre o estilo democrático e o uso problemático das redes sociais.

Relativamente ao neuroticismo como variável mediadora, foi possível concluir que o neuroticismo assume uma mediação total negativa na associação entre o estilo parental democrático e o uso problemático das redes sociais. Neste sentido, observou-se que o estilo parental democrático prediz negativamente o neuroticismo e que, por sua vez, o neuroticismo potencia o uso problemático das redes sociais. Desta forma, o neuroticismo evidencia-se como um mediador negativo na associação entre os estilos parentais positivos e o uso problemático das redes sociais. Estes resultados vão ao encontro do esperado na medida em que um ambiente parental responsivo e disponível constitui-se como um fator protetor no desenvolvimento de traços de personalidade emocionalmente ajustados. Desta feita, o traço de neuroticismo parece constituir-se como fator de risco no desenvolvimento de comportamentos do uso problemático das redes sociais.

Por outro lado, os estilos parentais negativos (estilo autoritário e permissivo) predizem positivamente o neuroticismo e este medeia parcialmente de forma positiva o desenvolvimento de comportamentos de uso problemático das redes sociais. Estes últimos resultados vão ao encontro do que seria expectável, na medida em que demostram novamente a relação positiva entre um ambiente parental 
negativo e o desenvolvimento de características negativas da personalidade que refletem instabilidade emocional sendo, por isso, estes indivíduos mais vulneráveis ao uso problemático das redes sociais.

Face aos resultados obtidos, considera-se que uma personalidade emocionalmente ajustada em conjunto com o estilo democrático contribui para menores níveis de uso problemático das redes sociais, visto que os indivíduos que apresentam essas características apresentam uma maior probabilidade de desenvolver autocontrolo nos seus comportamentos e menores dificuldades de interação com os outros. Por seu turno, a personalidade negativa em conjunto com estilos parentais negativos contribui para maior predisposição de uso problemático por adolescentes e jovens adultos.

\section{Implicações práticas, limitações e pistas futuras}

0 presente estudo assume um contributo positivo na compreensão da importância do estilo parental democrático adotado pelos cuidadores no desenvolvimento de comportamentos menos problemáticos no uso das redes sociais por adolescentes e jovens adultos. Por sua vez, permitiu ainda perceber que as características da personalidade assumem um papel relevante na associação entre os estilos parentais e o uso problemático das redes sociais. Neste sentido, espera-se que os resultados do presente estudo possam contribuir para um diagnóstico mais apropriado e uma melhor orientação terapêutica. Por outro lado, os dados suportam a necessidade de desenvolver programas de prevenção e intervenção nas escolas destinados aos jovens com o propósito de promover educação emocional e alertar para os riscos associados ao uso problemático e ainda a promoção de competências parentais com o objetivo de consciencializar para a importância da relação com os filhos.

Acrescem-se ao presente estudo algumas limitações, nomeadamente, o recurso a instrumentos de autorrelato passíveis de enviesamentos e o facto de a investigação ser de cariz transversal, não possibilitando analisar a comparação dos resultados ao longo do tempo nem o estabelecimento de relações de causalidade. Em termos de pistas futuras, seria importante abordar outras variáveis como, por exemplo, a resiliência e abranger outras áreas geográficas de Portugal de forma a tornar a amostra representativa da população geral.

\section{REFERÊNCIAS}

Água, J., Patrão, I., \& Leal, I. (2018). Relação dos traços de personalidade com a dependência ao smartphone. In Actas do $12^{\circ}$ Congresso Nacional de Psicologia da Saúde (pp. 429-437). ISPA-Instituto Universitário.

Amichai-Hamburger, Y., \& Vinitzky, G. (2010). Social network use and personality. Computers in Human Behavior, 26, 1289-1295. https://doi.org/10.1016/ j.chb.2010.03.018

Anaya, B., \& Pérez-Edgar, K. (2018). Personality development in the context of individual traits and parenting dynamics. Journal of Adolescence, 32, 1189-1207. https://doi.org/10.1016/j.newideapsych.2018.03.002

Assunção, R., \& Matos, P. M. (2014). Perspetivas dos adolescentes sobre o uso do Facebook: Um estudo qualitativo. Psicologia em Estudo, 19(3), 539-547. http://dx.doi.org/10.1590/1413-73722133716

Assunção, R., Costa, P., Tagliabue, S., \& Matos, P. (2017). Problematic facebook use in adolescents: associations with parental attachment and alienation to peers. Journal of Child and Family Studies, 26(11), 2990-2998. https://doi.org/10.1007/s10826-017-08172

Baumrind, D. (1967). Child care practices anteceding three patterns of preschool behavior. Genetic psychology monographs, 75(1), 43-88.

Baumrind, D. (1991). Effective parenting during the early adolescent transition. Family transitions, $2(1), 1$.

Bertoquini, V., \& Pais-Ribeiro, J. L. (2006). Estudo de formas muito reduzidas do modelo dos cinco fatores da personalidade. Psychologica, 43, 193-210.

Boubeta, A. R., Ferreiro, S. G., Salgado, P. G., \& Couto, C. B. (2015). Variables asociadas al uso problemático de internet entre adolescentes. Health and Addictions/Salud y Drogas, 15(1), 25-38. http://dx.doi.org/10.21134/haaj.v15i1.223

Bowlby, J. (1969). Attachment and loss (Vol.1: Attachment). Basic Books.

Chou, C., \& Lee, Y. (2017). The moderating effects of internet parenting styles on the relationship between Internet parenting behavior, Internet expectancy, and Internet addiction tendency. The Asia-Pacific Education Researcher, 26(3-4), 137-146. http://dx.doi.org/10.1007/s40299-017-0334-5

Dogan, H., Bozgeyikli, H. \& Bozdas, C. (2015). Perceived parenting styles as predictor of internet addiction in adolescence. International Journal of Research in Education and Science, 1(2), 167-174.

Giota, K., \& Kleftaras, G. (2013). The role of personality and depression in problematic use of social networking sites in Greece. Cyberpsychology: Journal of Psychosocial Research on Cyberspace, $7(3)$. http://dx.doi.org/10.5817/CP2013-3-6 
Gosling, S. D., Augustine, A. A., Vazire, S., Holtzman, N., \& Gaddis, S. (2011). Manifestations of personality in online social networks: Self-reported Facebook related behaviors and observable profile information. Cyberpsychology, Behavior, and Social Networking, 14, 483-488. https://doi.org/10.1089/cyber.2010.0087

John, O., \& Srivastava, S. (1999). The Big Five trait taxonomy: History, measurement, and theoretical perspectives. In L. Pervin \& O. John (Eds.), Handbook of personality: Theory and research (pp. 102138). Guilford Press.

Karl, K., Peluchette, J., \& Schlaegel, C. (2010). Who's posting facebook faux pas? A cross-cultural examination of personality differences. Inter- national Journal of Selection and Assessment, 18, 174-186. https://doi.org/10.1111/j.1468-2389.2010.00499.x

Leung, L., \& Lee, P. S. (2012). The influences of information literacy, internet addiction and parenting styles on internet risks. New Media \& Society, 14(1), 117-136. https://doi.org/10.1177/1461444811410406

Lo, J. (2019). Exploring the buffer effect of receiving social support on lonely and emotionally unstable social networking users. Computers in Human Behavior, 90, 103-116. https://doi.org/10.1016/j.chb.2018.08.052

Maddahi, M., Javidi, N., Samadzadeh, M., \& Amini, M. (2012). The study of relationship between parenting styles and personality dimensions in sample of college students. Indian Journal of Science and Technology, 5(9), 3332-3335.

Marôco, J. (2018). Análise estatística com o SPSS Statistics (7th ed.). ReportNumber.

Marôco, J. (2014). Análise de equações estruturais (2nd ed.). ReportNumber.

McKinney, B. C., Kelly, L., \& Duran, R. L. (2012). Narcissism or openness?: College students' use of facebook and twitter. Communication Research Reports, 29(2), 108-118. https://doi.org/10.1080/08824096.2012.666919

Mondin, E. (2017). Práticas educativas parentais e seus efeitos na criação dos filhos. Psicologia Argumento, 26(54), 233-244.

Pontes, H., \& Griffiths, M. (2015). The role of age, age of internet access initiation, and time spent online in the etiology of Internet addiction. Journal of Behavioral Addictions, 4(1), 30-31.

Pontes, H., Caplan, S., \& Griffiths, M. (2016). Psychometric validation of the generalized problematic internet use scale 2 in a Portuguese sample. Computers in Human Behavior, 63, 823-833. https://doi.org/10.1016/j.chb.2016.06.015

Pornsakulvanich, V. (2017). Personality, attitudes, social influences, and social networking site usage predicting online social support. Computers in Human Behavior, 76, 255-262. https://doi.org/10.1016/j.chb.2017.07.021

Rinhel-Silva, C. M., Constantino, E. P., \& Rondini, C. A. (2012). Família, adolescência e estilos parentais. Estudos de Psicologia, 29(2), 221-230. http://doi.org/10.1590/S0103-166X2012000200008

Robinson, C., Mandleco, B., Olsen, F., \& Hart, H. (1996, August, 12-16). Psychometric support for a new measure of authoritative, authoritarian, and permissive parenting practices: Cross-cultural connections. [Paper presentation in symposium: New measures of parental child-rearing practices developed in different cultural contexts]. XIVth Biennial International Society for the Study of Behavioral Development Conference, Quebec City, Canada.

Ross, C., Orr, E. S., Sisic, M., Arseneault, J. M., Simmering, M. G., \& Orr, R. R. (2009). Personality and motivations associated with facebook use. Computers in Human Behavior, 25(2), 578-586. https://doi.org/10.1016/j.chb.2008.12.024

Schofield, J., Conger. D., Donnellan, B., Jochem, R., Widaman, F., \& Conger, J. (2012). Parent personality and positive parenting as predictors of positive adolescent personality development over time. MerrillPalmer Quarterly, 58(2). 255-283. https://doi.org/10.1353/mpq.2012.0008

Secades, R., Calafat, A., Fernández, J.R., Juán, M., Duc, M., Skärstrand, E., Becoña, E. y Talic, S. (2014). Duration of internet use and adverse psychosocial effects among European adolescents. Adicciones, 26, 247253.

Song, H., Zmyslinski-Seelig, A., Kim, J., Drent, A., Victor, A., Omori, K., et al. (2014). Does facebook make you lonely?: A meta-analysis. Computers in Human Behavior, 36, 446-452. https://doi.org/10.1016/j.chb.2014.04.011

Tur-Porcar, A. (2017). Parenting styles and internet use. Psychology \& Marketing, 34(11), 1016-1022. https://doi.org/10.1002/mar.21040

Vlachopoulou, E., \& Boutsouki, C. (2014). Facebook usage among teenagers-the effect of personality and peer group pressure; an exploratory study in Greece. International Journal of Internet Marketing and Advertising, 8(4), 285-299. https://doi.org/10.1504/ijima.2014.067661 
Wilson, K., Fornasier, S., \& White, K. M. (2009). Psychological predictors of young adults' use of social networking sites. Cyber psychology, Behavior, and Social Networking, 13(2), 173-177. https://doi.org/10.1089/cyber.2009.0094

Yazdani, S., \& Daryei, G. (2016). Parenting styles and psychosocial adjustment of gifted and normal adolescents. Pacific Science Review B: Humanities and Social Sciences, 2(3), 100-105. https://doi.org/10.1016/j.psrb.2016.09.019

Zhou, Y., Li, D., Li, X., Wang, Y., \& Zhao, L. (2017). Big five personality and adolescent Internet addiction: The mediating role of coping style. Addictive Behaviors, 64, 42-48. https://doi.org/10.1016/j.addbeh.2016.08.009

$\begin{array}{ll}\text { Historial do artigo } & \\ \text { Recebido } & 03 / 2020 \\ \text { Aceite } & 03 / 2021 \\ \text { Publicado online } & 04 / 2021 \\ \text { Publicado } & 06 / 2021\end{array}$

